\title{
AN ALTERNATE STATISTICAL INTERPRETATION OF THE STRENGTH OF SNOW
}

\author{
By H. Gubler \\ (Eidg. Institut für Schnee- und Lawinenforschung, 726o Weissfluhjoch/Davos, Switzerland)
}

\begin{abstract}
Anstract. The basic features of the statistical models to describe brittle and ductile strength of snow are outlined and discussed. The concept of the "fundamental unit" is introduced. The models are applied to estimate the development of slab stability during heavy snowfalls and to simulate measurements of the tensile paper are compared with Sommerfeld's appling as a function of the load rate. The models developed in this in the starting zone of a slab avalanche.

RÉsumé. Modèles statistiques pour caractériser la résistance à la rupture de la neige. Les propriétés fondamentales de quelques modèles statistiques qui caractérisent la résistance ductile et cassante à la rupture de la neige sont décrites et discutées. L'idée des unités élémentaires est introduite. A partir de ces modèles on estime le développement de la stabilité dans la zone de décrochement d'une plaque de neige pendant une importante chute de neige. De même, la résistance de la neige à la traction qui est mesurée avec une centrifugeuse est simulée en fonction de la vitesse d'application de la charge. Les modèles décrits sont comparés à ceux que Sommerfeld a utilisés pour déterminer la résistance dans la zone de décrochement de plaques de neige.

Zusammenfassung. Statistische Interpretation der Festigkeit von Schnee. Die grundlegenden Eigenschaften von statistischen Modellen zur Beschreibung der Spröd- und Zähbruchfestigkeit von Schnee werden beschrieben und diskutiert. Das Konzept der Elementareinheit wird eingeführt. Aufgrund der Modelle werden die Stabilitätsentwicklung im Anrissgebiet einer Schneebrettlawine während eines Grosschneefalles sowie die Messung der Zugfestigkeit von Schnee mittels einer Zentrifuge als Funktion der Spannungsgeschwindigkeit simuliert. Die beschriebenen Modelle werden mit Sommerfeld's Anwendung statistischer Methoden zur Bestimmung der Festigkeit in Anrissgebieten von Schneebrettlawinen verglichen.
\end{abstract}

\section{INTRODUGTION}

In different papers Sommerfeld (1973, 1974, 1976) proposes statistical methods to extrapolate from sampled tensile and shear strengths to the tensile strength at the crown and the shear strength in the bed of a slab avalanche. In this paper the influence of the type of distribution used to describe the sample strength on the extrapolation to the snow strength is discussed. The concept of "fundamental units" instead of the macroscopic samples is introduced. The statistical models are modified considering the fundamental units instead of the macroscopic samples. The results are compared with Sommerfeld's conclusions. The stability development of a snow slab during a heavy snowfall and the measurement of tensile strength using the centrifugal method are simulated on the basis of the models introduced.

\section{DEFINITION OF THE FUNDAMENTAL unit}

Snow can be considered as a network of grains connected by ice bonds. At least in lowdensity snow, there does not exist any structural order of periodicity. Nevertheless the models discussed in this paper propose that the mechanical properties of snow can be traced back to the features of fundamental units. The viscoelastic properties of snow have been described on the basis of a similar model by Kry ( $1975[\mathrm{a}]$, [b]).

The basic features of the fundamental units are: (a) The snow may be subdivided in fundamental units. (b) Each fundamental unit acts as a force-conducting element in the snow. (c) As regards their positions, the properties of the fundamental units are distributed at random. (d) The properties (e.g. strength) of the fundamental units are defined by a distribution. (e) The properties of a distinct snow type may be calculated as a function of the density distribution of the corresponding features among the fundamental units, of the number density of the fundamental units, and the stress- or strain-rate. 
The basic elements of the fundamental units are proposed to be chains of grains. A chain is defined as a series of connected grains, transmitting a single force, terminated either by a structural element of higher connectivity or by a branch to other chains. The fundamental unit may be reinforced by additional parallel structural elements of higher connectivity (Kry, I975[a]; Gubler, 1978).

\section{DESGRIPTION OF BRITTLE STRENGTH BY EXTREME-VALUE STATISTICS}

The following concept holds for macroscopic samples defined as elementary forceconducting links (Sommerfeld, r 973) as well as for the fundamental units as defined above.

(a) If the body is loaded, the initial fracture of one link propagates elastically through the whole body and leads immediately to its fracture. The load rate has to be high enough to avoid mechanical relaxation or a rearrangement of the forces between the surviving links.

(b) Crack healing is impossible.

(c) The applied stress field is homogeneous; all weak links are equally stressed.

In general assumption (c) will not be fulfilled. Knowing the stress distribution among the links, proposition (c) may be replaced by a modification of the integral probability for the fracture of a single link $F(\sigma)$. With the strength distribution $f(s)$ of the separated links of the specimen under consideration, the integral probability $F(\sigma)$ that any separated link fractures as the load increases to $\sigma$ (proposition (c) fulfilled) results in

$$
F(\sigma)=\int_{0}^{\infty} f(s) \mathrm{d} s .
$$

If condition (c) is replaced by a density distribution $g(\sigma, x)$ of the link stresses (where $\sigma$ is the mean stress and $x$ an independent variable) $F(\sigma)$ is modified to

$$
F(\sigma)=\int_{s=0}^{\sigma} f(s)\left(\int_{x=s}^{\infty} g(\sigma, x) \mathrm{d} x\right) \mathrm{d} s .
$$

For the following calculations proposition (c) is considered to be fulfilled. This implies that the function $g$ is the Dirac $\delta$-function

$$
g(\sigma, x)=\delta(\sigma-x) .
$$

$\mathrm{I}-F(\sigma)$ is the probability that a separated link survives a load increase to $\sigma$. The probability that the $n$ weak links of a body survive a load $\sigma$ is given by

$$
\bar{P}(\sigma)=(\mathrm{I}-F(\sigma))^{n} .
$$

The corresponding probability density for the strength is:

$$
p(\sigma)=\frac{\mathrm{d}}{\mathrm{d} \sigma}\left[\mathrm{I}-(\mathrm{I}-F(\sigma))^{n}\right] .
$$

The most probable strength $\sigma_{\mathrm{m}}$ of a body with $n$ weak links results from the maximum condition:

$$
\frac{\mathrm{d} p\left(\sigma_{\mathrm{m}}\right)}{\mathrm{d} \sigma}=\mathrm{o} .
$$

From Equations (I), (3), (4), and (5) it follows that

$$
\frac{\mathrm{d} f\left(\sigma_{\mathrm{m}}\right)}{\mathrm{d} \sigma}\left[\mathrm{I}-F\left(\sigma_{\mathrm{m}}\right)\right]-f^{2}\left(\sigma_{\mathrm{m}}\right)(n-\mathrm{I})=0 .
$$


The variance $\mu$ of the body-strength distribution is given by the second moment of $p(\sigma)$ relative to the mean value $\sigma_{0}$ of $\sigma$ (first moment):

$$
\begin{aligned}
\mu & =\int_{0}^{\infty}\left(\sigma-\sigma_{0}\right)^{2} p(\sigma) \mathrm{d} \sigma \\
& =\int_{0}^{\infty}\left(\sigma-\sigma_{0}\right)^{2} n f(\sigma)(\mathrm{I}-F(\sigma))^{n-1} \mathrm{~d} \sigma,
\end{aligned}
$$

where $\sigma_{0}$ is given by

$$
\begin{aligned}
\sigma_{0} & =\int_{0}^{\infty} \sigma p(\sigma) \mathrm{d} \sigma \\
& =\int_{0}^{\infty} \sigma n f(\sigma)(\mathrm{I}-F(\sigma))^{n-1} \mathrm{~d} \sigma .
\end{aligned}
$$

The lower limits of all integrals with respect to $\sigma$ have to be set equal to o and not as usual $-\infty$ to avoid integration of negative strength values. This condition is very important for link strength distribution functions which are not zero for negative strengths as, e.g. the normal distribution would be.

To be able to calculate $p(\sigma)$ and the ratio of the most probable body fracture strength $\sigma_{\mathrm{m}}$ to the most probable link fracture strength $s_{\mathrm{m}}$ as a function of $n$ for any distribution function $f(s)$, a desk-calculator program was written which solves the equations by numerical methods and plots the results: $f(s), p(\sigma, n),\left[\sigma_{\mathrm{m}} / s_{\mathrm{m}}\right](n)$.

\section{DEFinition AND FEATURES OF THE INVESTIGATEd LINK STRENGTH DisTRibUtions $f(s)$}

\section{Weibull distribution}

$$
\left.\begin{array}{ll}
f(s)=r\left[\frac{s-s_{\mathrm{u}}}{\Delta}\right]^{r-\mathrm{I}} \exp -\left[\frac{s-s_{\mathrm{u}}}{\Delta}\right]^{r} & \text { for } s \geqslant s_{\mathrm{u}}, \\
f(s)=0 & \text { for } s<s_{\mathrm{u}} .
\end{array}\right\}
$$

Apparently Weibull (Weibull, I939[a], [b]) chose this distribution for mathematical tractability. This distribution proposes the existence of a minimal link strength. There are no links with zero or almost zero strength. $r, s_{\mathrm{u}}, \Delta$ are the distribution parameters. The most probable link strength is given by the relation

$$
s_{\mathrm{m}}=\Delta\left[\frac{|r-\mathrm{I}|}{r}\right]^{1 / r}+s_{\mathrm{u}} .
$$

The most probable value of $p(\sigma)$ decreases as $n^{-1 / r}$, the variance as $n^{-2 / r}$ (Epstein, 1948).

2. Normal distribution

$$
\left.\begin{array}{ll}
f(s)=\frac{\mathrm{I}}{(2 \pi)^{\frac{1}{2}} \Delta} \exp -\frac{\mathrm{I}}{2}\left[\frac{s-s_{0}}{\Delta}\right]^{2} & \text { for } s \geqslant 0, \\
f(s)=0 & \text { for } s<0 .
\end{array}\right\}
$$

To avoid finite probabilities for negative strength, $f(s)$ is set equal zero for $s<0$. But it retains a finite probability for zero link strength.

$\sigma_{\mathrm{m}}$ decreases as $(\ln n)^{\frac{1}{2}}$, the variance of $p(\sigma)$ is given by $\pi^{2} \sigma^{2} / \mathrm{I} 2 \log n$ (Epstein, I948). 
3. Log-normal distribution

$$
f(s)=\frac{\mathrm{I}}{(2 \pi)^{\frac{1}{2}} \ln \Delta} \exp -\frac{\mathrm{I}}{2}\left[\frac{\ln s-\ln s_{0}}{\ln \Delta}\right]^{2} .
$$

This type of distribution is known to be typical for stereological parameters such as, e.g. particle-size distributions. The probability for zero strength is zero, but is finite for any $s>0$.

\section{Discussion OF THE NUMERIGAL GOMPUTATIONS}

$p(\sigma)$ and the ratio $\sigma_{\mathrm{m}} / s_{\mathrm{m}}$ were calculated as functions of $n . s$ and $\Delta$ were normalized to $s_{\mathrm{m}}$ (the most probable value of $s$ ). The range of $n$ is $\mathrm{I}$ to $10^{5}$, the range of $s / s_{\mathrm{m}}$ o to 2 , the relative deviation $\Delta / s_{\mathrm{m}}$ is 0.2 . Using the Weibull distribution, $\Delta / s_{\mathrm{m}}$ is multiplied by about $\mathrm{I} .7$ to get similar widths as for the normal distributions. For the Weibull distributions, $r$ is set equal to $\mathrm{I} .6, s_{\mathrm{m}}=\mathrm{I}$. In Figures $\mathrm{I}-3$ the weak-link strength distributions $f(s)$ are plotted with

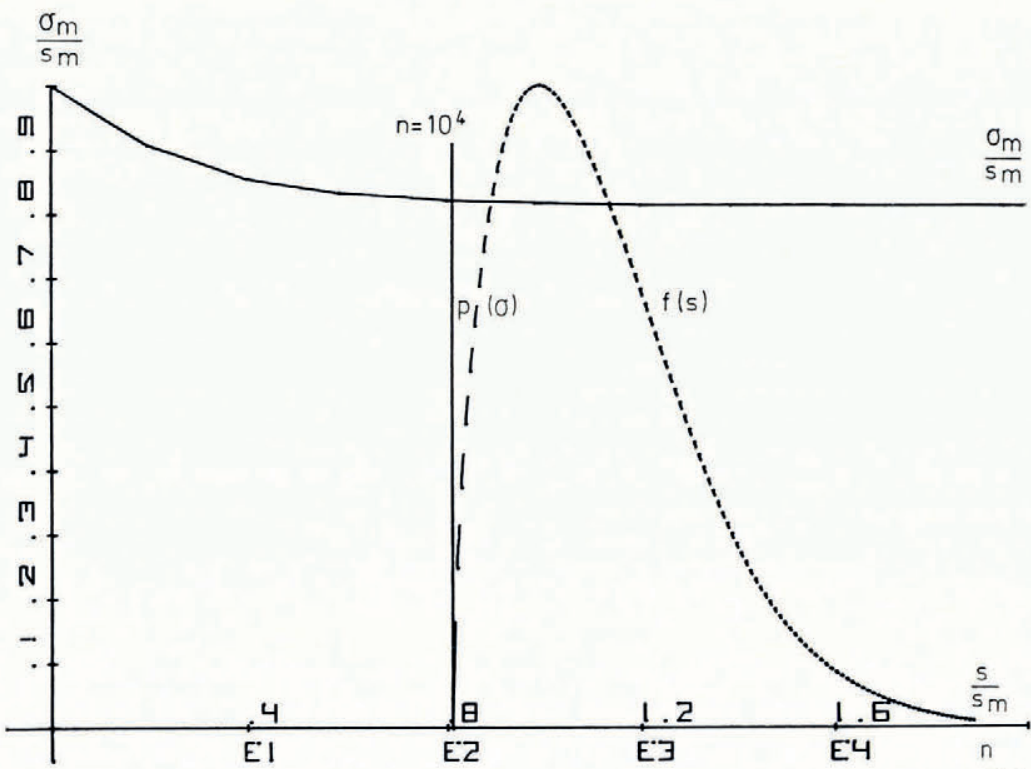

Fig. 1 . Brittle strength model: Ratio of brittle strength of snow $\sigma_{\mathrm{m}}$ to the mean strength of the separated fundamental units: for a Weibull type of distribution of strength, - - - as a function of the number of fundamental units of the test volume. $\Delta / s_{\mathrm{m}}=0.2, p(\sigma)$ is plotted for $n=10^{4}$.

broken curves, the body strength distributions $p(\sigma)$ and the ratio $\sigma_{\mathrm{m}} / s_{\mathrm{m}}$ (starting at I.o) with full curves. Only the Weibull distribution (Fig. I) shows an almost constant ratio of body to link strength for high $n$ whose limiting value is determined by the parameters $r$ and $s_{\mathrm{u}}$. This result implies the existence of a critical volume of a specimen, above which the body strength would be almost independent of further volume increase. This behaviour follows directly from the definition of a minimum strength. For small $r(\approx \mathrm{I})$ the distributions are very asymetric (steep rises from $s_{\mathrm{u}}$ to $s_{\mathrm{m}}$ ). For constant $\Delta / s_{\mathrm{m}}$ the asymptotic value of $\sigma_{\mathrm{m}} / s_{\mathrm{m}}$ decreases with increasing $r$.

For the log-normal distributions (Fig. 2) with comparable deviations $\sigma_{\mathrm{m}} / s_{\mathrm{m}}$ never reaches a constant value $>0$ independent of $n$ (for $n \rightarrow \infty, \sigma_{\mathrm{m}} / s_{\mathrm{m}} \rightarrow 0$ ), but the decrease for high $n$ is quite slow. For the normal distribution (Fig. 3) $\sigma_{\mathrm{m}} / s_{\mathrm{m}}$ already equals zero for finite $n$. 


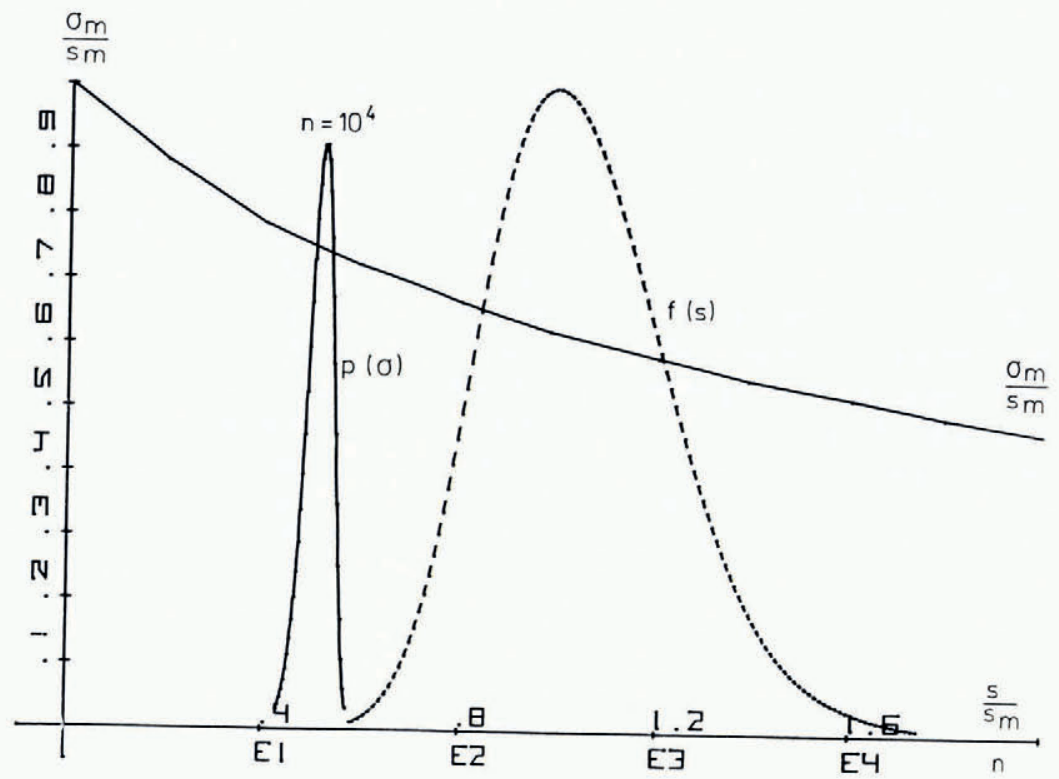

Fig. 2. Brittle strength model: Equivalent conditions as for Figure I but for log-normal distribution of strength of the separated fundamental units.

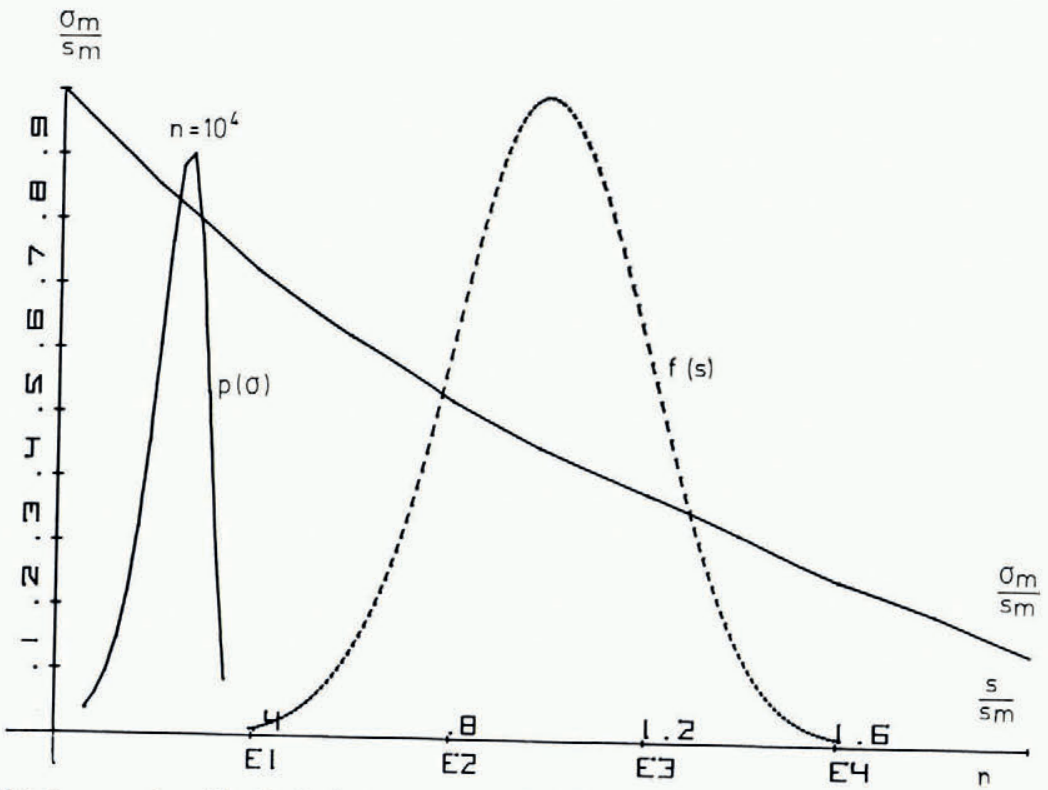

Fig. 3. Brittle strength model: Equivalent conditions as for Figure I but for a normal distribution for the strength. 


\section{Application of Smallest-value statistics to snow}

The magnitude of the minimal strain-rate in medium-density snow to initiate a brittle fracture may be estimated from different investigations (Salm, 197 1, Gubler, 1976). It turns out that dissipative processes are negligible only for strain rates $\dot{\epsilon} \geqslant \mathrm{I} \mathrm{s}^{-1}$. The strain-rates involved in natural releases of slab avalanches are smaller by at least five orders of magnitude. Therefore the natural initiation of primary fractures in starting zones of slab avalanches cannot be described with the model under consideration. Even pressure waves originating from detonations $\left(\dot{\epsilon} \approx 1 \mathrm{O}^{-3} \mathrm{~s}^{-1}\right)$ do not initiate perfectly brittle fractures. Only the secondary fracture spreading out from the primary fracture volume is of the brittle type. The propagation velocity of the secondary fracture is of the same order of magnitude as the propagation velocity of the stress wave (approximately the velocity of sound), therefore relaxation processes at the crack front seem to be impossible. The highly stressed volume of snow at the front of the crack which has to be considered for an estimation of the number of stressed fundamental units $n$ will be quite small $\left(n \approx \mathrm{IO}^{4}\right.$ to $\mathrm{IO}^{5}$, corresponding to a small number of rows of fundamental units in front of the propagating crack). Estimates of the relative width of the density distribution $f(s)$ of the strengths of the fundamental units result in values of $(10-30) \%$ (Kry, 1975). Various reasons favour a log-normal type for the strength distribution (which is the type of distribution of bond and grain diameters in sintered materials). These propositions lead to ratios of the most probable brittle strength of snow at the front of the propagating secondary fracture to the mean strength of the separated fundamental units of 0.35 to 0.8 .

\section{A statistical model to estimate dugtile strength}

\section{Basic features of the model}

Daniels (1945) developed a model to describe the strength of a bundle of threads. The main performance of this model is given by the fact that during loading a stress rearrangement from fractured threads to the surviving stronger threads is possible. A small generalization leads to the following concept:

(a) If a load is applied slowly, mechanical relaxation causes equal stress for every link. If the load is increased further, the weakest of the equally stressed links fractures and the total load is redistributed among the remaining links. The load increase has to be slow enough to allow a uniform stress distribution between all surviving links at any time.

(b) Body strength is reached when a redistribution of the stresses is no longer possible.

(c) The healing of fractured links is not possible.

Condition (a) may be slightly modified by requiring a defined stress distribution among the surviving links instead of equal stress for every link. As regards condition (c), in lowdensity snow, crack healing or the healing of broken bonds between the ice grains is not an important mechanism. In most cases the separation of the fracture surfaces impedes the healing of the broken bond. The growth of the existing bonds as well as the formation of new bonds which lead to strength increases of the fundamental units can be included by choosing a time-dependent strength distribution function for the fundamental units.

\section{Statistical treatment}

Knowing the strength distribution function $f(s)$ of the separated elements of the specimen, the number $n_{\sigma}$ of the surviving links per unit area with strengths larger than $\sigma$ (stress increased to $\sigma$ ) may be calculated. For equally stressed links it follows that 


$$
n_{\sigma}=n \int_{\sigma}^{\infty} f(s) \mathrm{d} s
$$

where $n$ is the total number of links per unit area before any fracture occurred.

The introduction of a density distribution $g(s, x)$ for the link stresses ( $s$ is the mean stress, $x$ the independent variable) modifies $n_{\sigma}$ to

$$
n_{\sigma}=n\left\{\mathrm{I}-\int_{s=0}^{\infty}\left(f(x) \int_{x=s}^{\infty} g(\sigma, x) \mathrm{d} x\right) \mathrm{d} s\right\} .
$$

With $g(s, x)=\delta(s-x)$, Equation (14) results again in Equation (13)

$$
n_{\sigma}=n\left\{\mathrm{I}-\int_{s=0}^{\infty}\left(f(x) \int_{x=s}^{\infty} \delta(\sigma-x) \mathrm{d} x\right) \mathrm{d} s\right\}=n \int_{\sigma}^{\infty} f(s) \mathrm{d} s .
$$

For the following calculations proposition (a) is considered to be fulfilled.

If the load per link is increased to $\sigma$, the $\left(n-n_{\sigma}\right)$ links with strength less than $\sigma$ are broken and the load $\Sigma$ per unit area is redistributed among the remaining $n_{\sigma}$ links:

$$
\Sigma=n_{\sigma} \sigma=\sigma n \int_{\sigma}^{\infty} f(s) \mathrm{d} s .
$$

The maximum possible load $\Sigma$, that is the strength of the specimen per unit area results from the condition:

$$
\frac{\mathrm{d} \Sigma_{\mathrm{m}}}{\mathrm{d} \sigma}=0 .
$$

To be able to compare the resulting specimen strength $\Sigma_{\mathrm{m}}$ with the most probable link strength $s_{\mathrm{m}}$ the quotient $\Sigma_{\mathrm{m}} / n s_{\mathrm{m}}$ is calculated for different link strength distributions and
widths:

$$
\frac{\Sigma_{\mathrm{m}}}{n s_{\mathrm{m}}}=\frac{\sigma_{\mathrm{m}}}{s_{\mathrm{m}}} \int_{\sigma_{\mathrm{m}}}^{\infty} f(s) \mathrm{d} s,
$$

where $\sigma_{\mathrm{m}}$ is the maximum mean link stress given by Equation (I6). This gives $\Sigma_{\mathrm{m}} / n s_{\mathrm{m}}$ as a function of the distribution width and type. The distribution $f(s)$ and the development of the quotient $\Sigma / n$ during the loading process are plotted with the help of a desk-calculator program.

\section{Discussion of the NUMERICAL GOMPUTATION}

$s$ and $\Delta$ were normalized to $s_{\mathrm{m}}$ (the most probable value of $s$ ). All distribution integral functions are normalized to one in the range from o to $\left(\mathrm{ro} \Delta / s_{0}\right)+\mathrm{I}$. The range of $s / s_{\mathrm{m}}$ is o to 3 . The relative deviations $\Delta / \sigma_{\mathrm{m}}$ vary from $0 . \mathrm{I}$ to 0.5 in steps of o.I. For the Weibull distribution $\Delta / \sigma_{\mathrm{m}}$ is multiplied by c. I.7 to get similar widths as for the log-normal distributions. For the Weibull distribution $m$ is set equal to r.6, $s_{\mathrm{m}}$ equals $\mathrm{r}$. In Figures 4 to 6 the link strength distributions $f(s)$ are plotted with dashed lines, the functions $\Sigma(\sigma)$ and the ratio $\Sigma_{\mathrm{m}} / s_{\mathrm{m}}$ with full lines. The ratios $\Sigma_{\mathrm{m}} / s_{\mathrm{m}}$ for the normal and the log-normal link-strength distribution show a similar dependence on $\Delta / s_{\mathrm{m}}$. They decrease from I (normal distribution) to a minimum value for $\Delta / s_{\mathrm{m}} \approx 0.5$ and increase afterwards again. For the normal distribution this increase is caused by the zero assumed for $f(s)$ for $s<0$. For the log-normal distribution the increase results from its asymmetric behaviour. For the Weibull distribution the decrease of $\Sigma_{\mathrm{m}} / s_{\mathrm{m}}$ as a function of $\Delta / s_{\mathrm{m}}$ is slower and in the interesting range of $\Delta / s_{\mathrm{m}}$ there is no re-increase. 


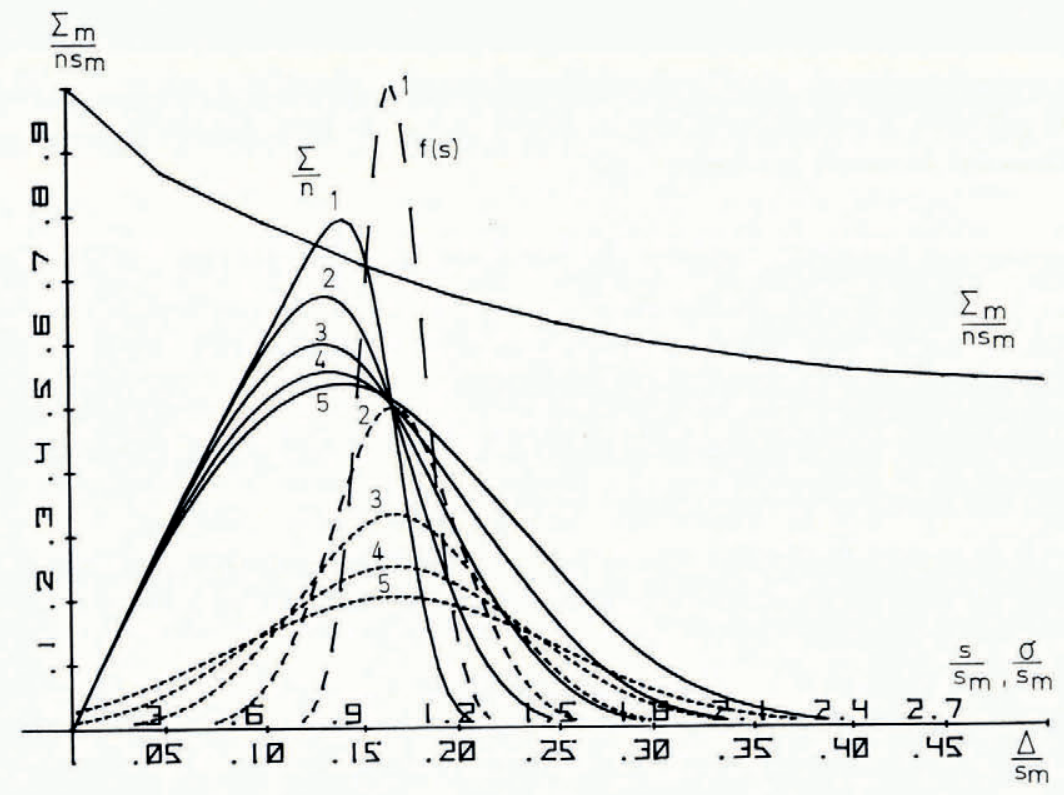

Fig. 4. Ductile strength model: Ratio of ductile strength of snow $\Sigma_{\mathrm{m}}$ to the mean strength of the fundamental units $s_{\mathrm{m}}:-\overline{\text { intion }}$ as a function of the relative width of the distribution of the strength of the fundamental units. $\Delta / s_{\mathrm{m}}$ for a normal distribution of strength

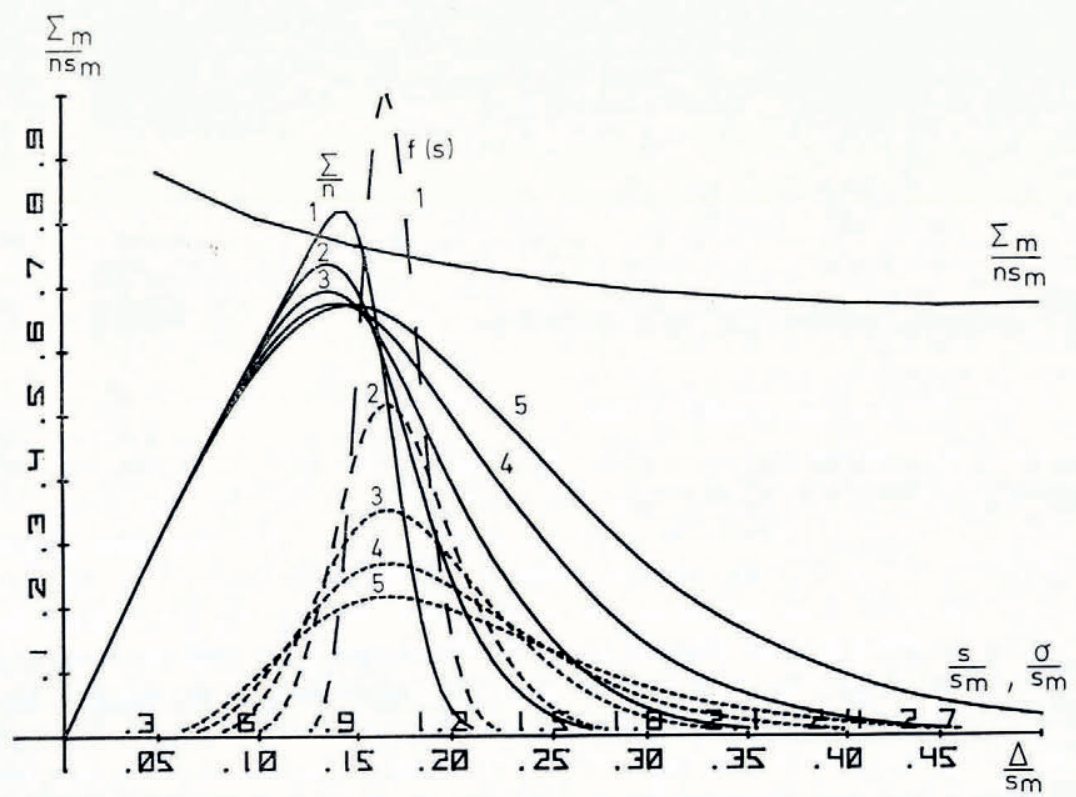

Fig. 5. Ductile strength model: Equivalent conditions as for Figure 4 but for log-normal type of distribution for the strength $f(s)$. 


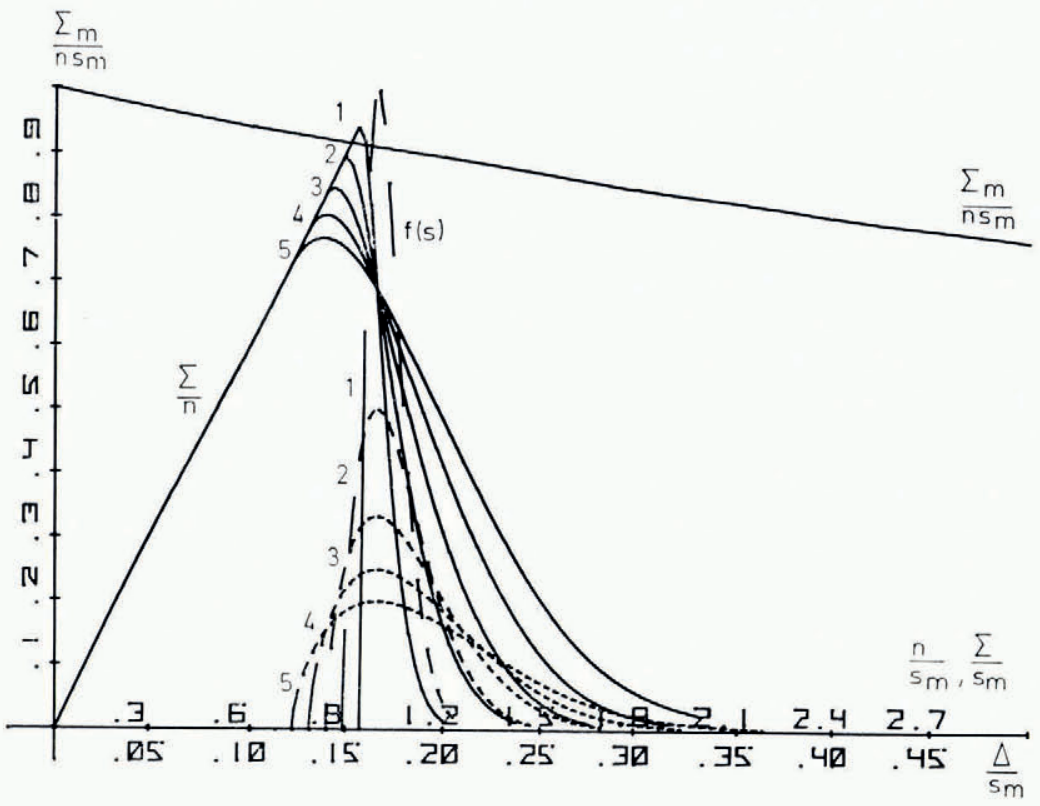

Fig. 6. Ductile strength model: Equivalent conditions as for Figure 4 but for a Weibull type of distribution for the strength $f(s)$.

\section{Application of the statistical treatment of Dugtile strength to SNOW}

The ductile type of strength is responsible for most natural and artificially-induced fractures in snow (Salm, I971; Gubler, 1976). The smaller the strain-rate $\dot{\epsilon}$ the better the redistribution of the loads among the surviving links, the smaller the remaining internal forces. The ductile strength depends only on the relative width of the link strength distribution and is independent of the macroscopic test volume. The density of the fundamental units for low-density snow is of the order of $\mathrm{IO}^{-10} \mathrm{~m}^{-3}$. The brittle strength of $\mathrm{I} \mathrm{m}^{3}$ of snow under these conditions amounts to one-fifth of the mean strength of the fundamental units $\left(\Delta / s_{\mathrm{m}}=0.3\right.$, log-normal type of strength distribution), whereas the corresponding ratio for the ductile strength is 0.7 . From this estimate a ratio of ductile to brittle strength of 3.5 follows. For the above calculation the strength of the fundamental units was considered to be independent of strain-rate.

Simulation of the PRIMARY FRAGTURE PROGESS OF A SLAB AVALANCHE USING A TIME-DEPENDENT DISTRIBUTION FUNGTION FOR THE STRENGTHS OF THE FUNDAMENTAL UNITS $f(s, t)$

The snow cover of a potential fracture zone of a slab avalanche is composed of sections of different stress states, stress magnitudes, and stabilities. The initial fracture will occur in the section with lowest stability relative to a ductile fracture. The section has to be small enough so that its macroscopic stress field can be considered to be homogeneous. We define this section as the primary fracture volume of the slab avalanche. Strength and stress in this primary fracture volume change continuously with time. The ductile fracture of this volume may propagate to the neighbouring sections of the snow cover (brittle fracture) and release the slab. The stability development in the primary fracture volume will now be investigated using a time-dependent strength distribution function $f(s, t)$.

To simulate a strength increase of the unbroken elements by sintering (constant temperature metamorphism) and by the formation of new bonds, the density distribution of the 
strengths of the fundamental units may be modified. For the following estimates the strength development in the slab bed is considered to be mainly responsible for the natural release of slab avalanches (Sommerfeld, r976).

To describe the strength increase as a function of time the following time dependences may be involved:

Sintering: $s \propto t^{2 / 5}$ (theoretical growth of bond cross-sections).

Settling: Probably has no effect on the thin lubricating layers. Density dependence of shear strength for low-density snow seems to be low.

Increase of normal pressure: For low shear strength and normal pressures in the range from zero to a maximum pressure less than the shear strength (new snow). A. Roch (Sommerfeld, I976) found an almost linear dependence of shear strength on normal pressure.

Figure 7 shows an example of the development of $\Sigma_{\mathrm{m}} / n$ as a function of a time-dependent log-normal link-strength distribution function. In the examples the relative deviation is kept constant. The most probable element strength $s_{\mathrm{m}}$ is increased from $\mathrm{I}$ to 2 either proportional to $t^{2 / 5}$ or $t$ within a time $t=0$ to $\mathrm{I}$.

Assuming a linear stress increase in time (such as provided by continuous snowfall for $24 \mathrm{~h}(t=\mathrm{I})$ starting at zero for $t=0$, avalanche (I) (Table I) starts after $5 \cdot \mathrm{I} \mathrm{h}$, avalanche (2) after I I.8 h, avalanche (3) after I4.I h, avalanche (4) after I $8 \mathrm{~h}$, avalanches (5) and (6) will not occur if precipitation stops after $24 \mathrm{~h}$.

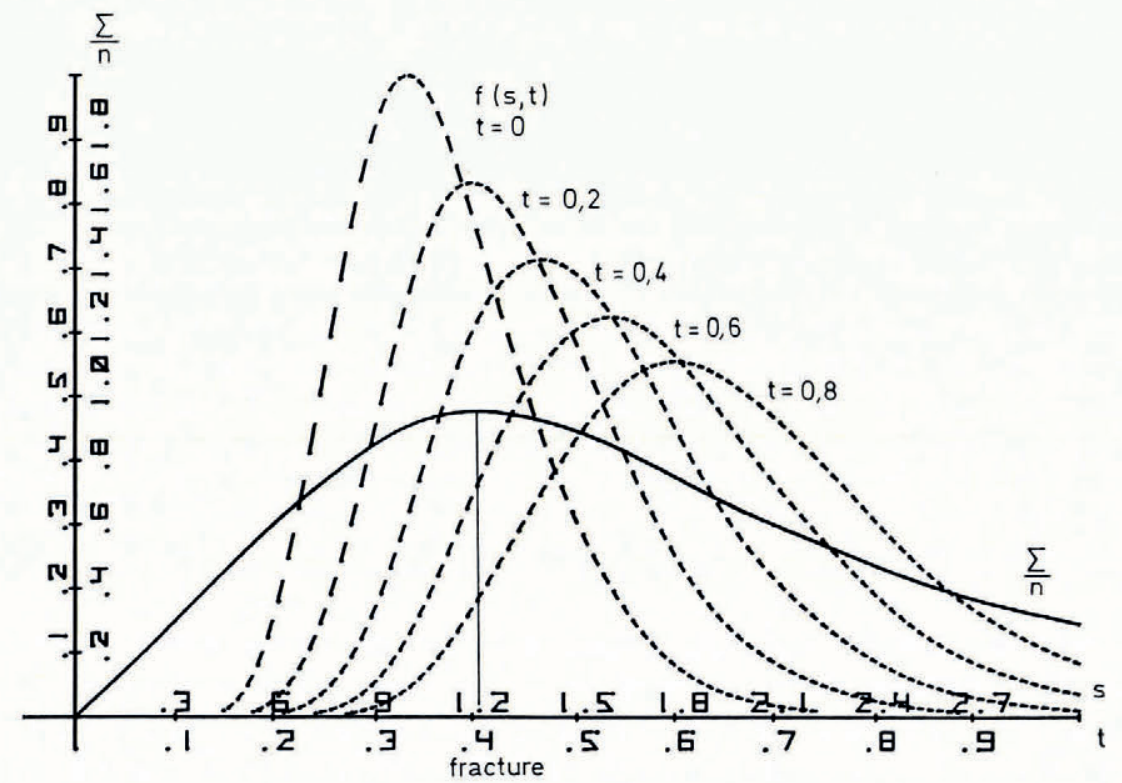

Fig. 7. Theoretical development of the reciprocal stability (ratio of stress to strength) for a linear increase of the mean strength of the fundamental units as a function of time and constant precipitation (example $I$ in Table I).

Table I. Time of failure as a function of the time dependence of $f(s, t)$ time of failure Strength 
These very theoretical estimates should show how important the exact determination of the relative deviation of the link-strength distribution and of the time dependence of the strength increase are.

If the conditions of the models described in this paper can be proved definitely by experiments, some additional conclusions may be drawn from the model calculations.

I. The higher the relative width of the strength distribution of the fundamental units, the higher the ratio of ductile to brittle strength, and the easier the spreading of the secondary fracture from a natural induced primary fracture.

2. The higher the relative width of the strength distribution of the fundamental units, the lower the probability of a natural avalanche release. But if a natural or artificially induced primary fracture occurs, the resulting low stability to secondary fracture may lead to enlarged slabs.

\section{Simulation OF BRitTle AND DUGTILE STRENGTH TESTS USING THE GENTRIFUGAL METHOD}

The brittle and ductile strength measurements of samples using the centrifugal method can be simulated on a desk calculator.

Because of the lack of exact information, the strength distributions of the fundamental units are considered to be independent of the stress rate. The integral probability of the sample strength is plotted as a function of the spinning frequency for brittle strength. The ductile strength is marked by a vertical line (Figs 8 and 9 ).

The calculation of the brittle strength of the sample is based on Equation (3):

$$
\bar{P}(\sigma)=(\mathrm{I}-F(\sigma))^{n}
$$

(for equally stressed fundamental units). $\bar{P}(\sigma)$ is the probability that a unit volume of the snow sample has a strength equal to $\sigma . n$ is defined as the number density of the fundamental units.

For the calculation of the sample strength in the stress field of a spinning cylinder, the sample cylinder is thought of as cut into equally thick slices. The probability that a slice of

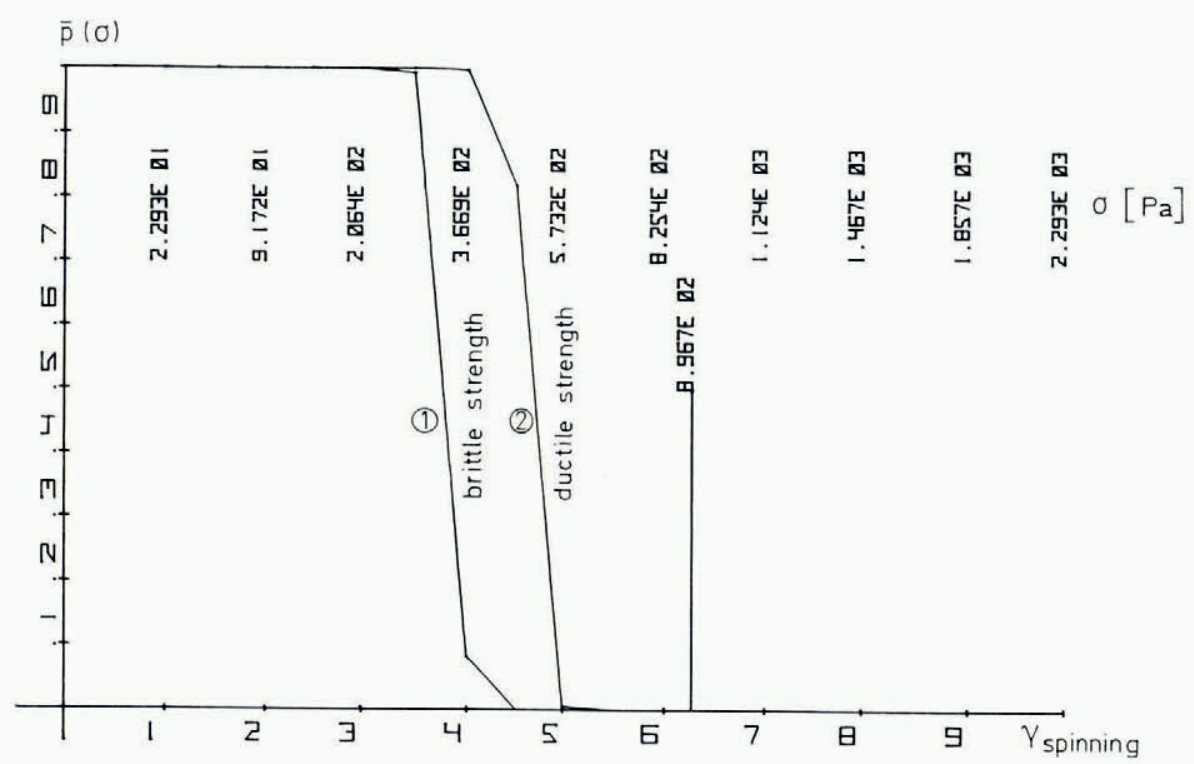

Fig. 8. Simulation of centrifugal tensile strength as a function of link density for two types of similar ductile strength and density. I: $n=10^{11} \mathrm{~m}^{-3}, s_{\mathrm{m}}=6 \times 10^{-5} \mathcal{N} ; 2: n=10^{8} \mathrm{~m}^{-3}, \mathrm{~s}_{\mathrm{m}}=6 \times 10^{-3} \mathcal{N}$. 


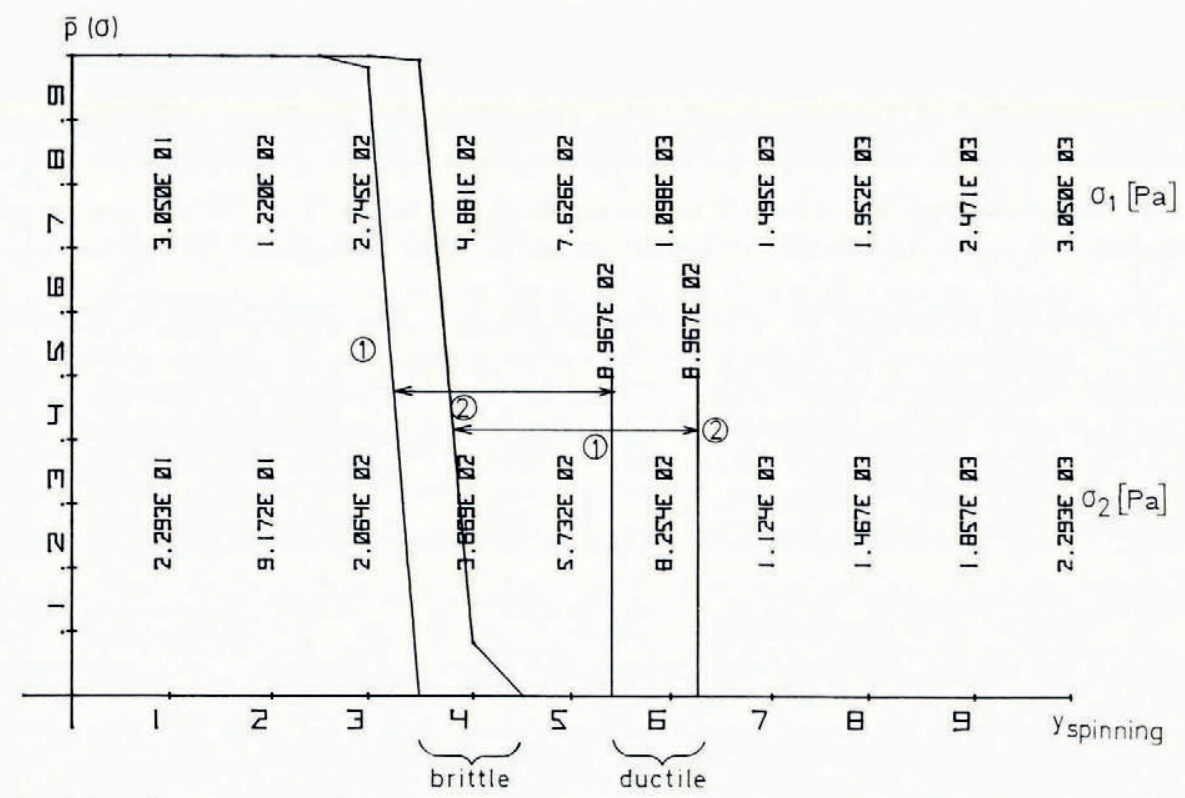

Fig. 9. Simulation of centrifugal tensile strength tests as a function of sample size. The ratio of the cross-sectional areas of sample 1 to sample 2 amounts to 4 . The corresponding ratios of ductile to brittle strength are: for the larger sample 2.9, for the smaller sample 2.6.

thickness $\Delta L$ at a mean distance $l$ from the spinning axis survives an increase of the spinning frequency to $\nu$ is given by

$$
\bar{P}(\nu, l)=\left\{\mathrm{I}-\int_{0}^{\sigma(l, v)} f(s) \mathrm{d} s\right\}^{n V_{\mathrm{s}}},
$$

where $n V_{\mathrm{s}}$ is the number of links in the slice $\left(=\frac{1}{4} \pi d^{2} n \Delta L\right) . \bar{P}(\nu)_{\mathrm{t}}$ for the whole sample results as the product of all $P(\nu, l)_{\mathrm{s}}$

$$
\bar{P}(\nu)=\left[\prod_{l=0}^{L}\left\{\mathrm{I}-\int_{0}^{\sigma(l, v)} f(s) \mathrm{d} s\right\}^{n V_{\mathrm{s}}}\right]^{2} .
$$

The result has to be squared to take both ends of the sample cylinder into account:

$$
\bar{P}(\nu)=\prod_{l=0}^{L}\left\{\mathrm{I}-\int_{0}^{\sigma(l, v)} f(s) \mathrm{d} s\right\}^{2 n V_{\mathrm{s}}} .
$$

For the calculation of the ductile strength, only the fundamental units in the mid-plane of the cylinder have to be considered (the number of the fundamental units in the cross-section has to be high enough to allow a statistical description). The resulting strength is well defined and the failure occurs in the mid-plane of the sample cylinder.

Figure 8 shows the dependence of the brittle strength on the number density of the fundamental units for two snow types of equivalent ductile strength. Figure 9 shows the dependence of the ratio of ductile to brittle strength on the sample size. In Figure ro measured 
data of the tensile strength are plotted as a function of the load rate. These experimental results show a significant increase of strength with decreasing stress-rate. The estimate of brittle and ductile strength on the base of the statistical models furnish only extreme values for the strengths.

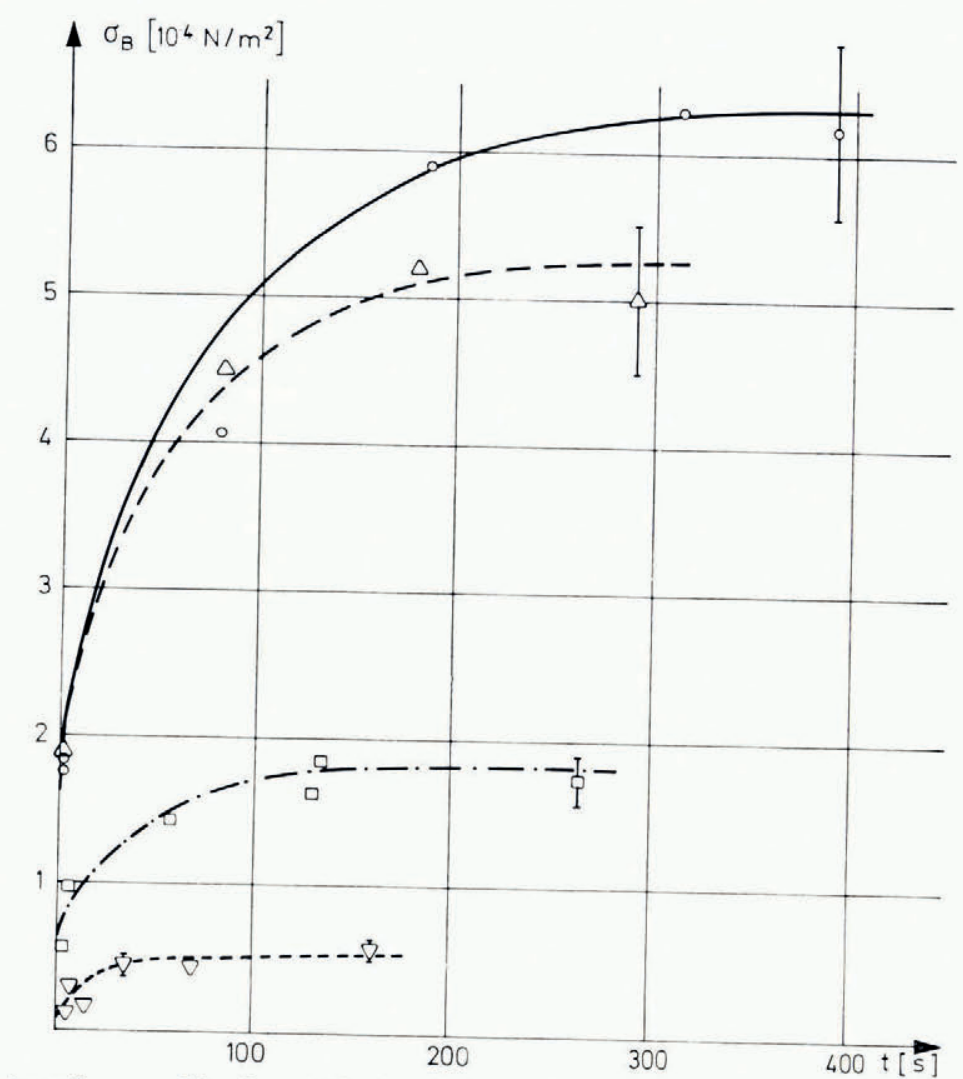

Fig. Io. Tensile strength measured by the centrifugal method as a function of the time between the start of the centrifuge and failure of the sample for different types of snow.

Discussion of SommerfeLd's Estimate on the Basis OF STATISTICAL MODELS FOR THE STRENGTHS

There are two very important differences in the application of the statistical models to snow in this paper and the estimate given by Sommerfeld (1973, 1974, 1976)

(a) Sommerfeld considers the measured sample strengths to be the link strengths.

(b) Sommerfeld uses the Weibull distribution fitted to the strength distribution of his tensile-strength measurements as the basis for the brittle model, and the normal distribution fitted to the strength distribution of the shear-strength measurements as the base for the ductile strength model.

Sommerfeld performed centrifugal tensile tests on $0.5 \times \mathrm{IO}^{-3} \mathrm{~m}^{3}$ and $2.3 \times \mathrm{IO}^{-3} \mathrm{~m}^{3}$ samples of cylindrical shape with diameters of $6 \times \mathrm{IO}^{-2}$ and $\mathrm{I} 2 \times \mathrm{IO}^{-2} \mathrm{~m}$. From the strength distribution of these samples he wanted to estimate the brittle tensile strength at the crown of a slab avalanche.

In the natural snow cover there may be large flaws produced by wind action during precipitation, by rocks, gliding, and settling. These large flaws led to zero or almost zero 
strength measurements. To eliminate the effect of large flaws Sommerfeld designed the larger $2.3 \times \mathrm{IO}^{-3} \mathrm{~m}^{3}$ sample tube. Nevertheless, to reduce systematic errors further, the three lowest strengths of each measured distribution were not used in the Weibull determination (Sommerfeld, 1974). In some cases the Weibull parameters were significantly changed by this data reduction but at the same time the fits were improved. Sommerfelds' calculations result in a snow strength (for volumes larger than $\mathrm{I} \mathrm{m}^{3}$ ) with an integral fracture probability of 0.999 of about I.I times the minimum sample strength. This strength ratio corresponds to a volume or link-number ratio of about 3 to 4 orders of magnitudes (Sommerfelds' critical volume being about $\mathrm{I} \mathrm{m}^{3}$ and the effective sample volume some $\left.10^{-4} \mathrm{~m}^{3}\right)$. His Weibull parameter $r$ changed from 0.9 to I.6. Different problems arise from Sommerfeld's sampling method: The natural strain-rate differs by some orders of magnitude from the test strain-rate. The samples have to be small enough to guarantee a homogeneous natural stress distribution over the sample size. It is likely that brittle tensile strength is only involved in the propagation of the secondary fracture. The additionally stressed volume at the front of the crack is probably of a comparable size to the sample volumes mentioned. The weak flaws defined above may occasionally act as primary fracture volumes. They cannot be considered to be distributed at random even relative to critical volumes of $\mathrm{I}^{3}$. From the strong dependence of the strength distribution on the sample size, it follows that the link definition of Sommerfeld is very critical. Sommerfeld found that brittle strength has to be volume independent for volumes larger than about $\mathrm{I}^{3}$. This volume independence characterizes the Weibull distribution. But because of the necessarily high strain-rates to produce brittle failure the volume to be considered in front of a brittle crack is limited by the propagating stress front and the stress distribution and will be much smaller than $\mathrm{I}^{3}$. If the sample strength of Sommerfeld is thought to be log-normally distributed, the resulting brittle strength will be significantly changed. Also the knowledge of the strong dependence of the sample strengths on the stress-rate shows that the more or less accidental definition of the link unit by Sommerfeld is not optimal. It seems to us that the arbitrary size of the test samples has to be replaced by some kind of fundamental units as defined at the beginning of this paper in order to obtain consistent results.

Sommerfeld proposes an application of the ductile strength model to describe the shear strength in the bed of a slab avalanche. He mentioned the following points to justify the application of the thread model: "During shear failure, the failing parts are in contact and friction and crack healing due to new bond formation impedes crack growth. For these reasons a quasi-failure is the likely initiating event. There is a strong possibility that a failure in a small part of the bed surface would not propagate elastically, and that the whole bed surface would not fail."

Sommerfeld considered the shear-frame area as the link unit. Shear-strength data measured by Perla were fitted to a normal distribution with a relative standard deviation of about 0.5 . On the basis of the ductile strength model he arrived at a shear strength in the slab bed reduced by a factor 0.53 compared with the mean shear-frame strength. He thought of this result as an explanation of the stability factor defined by Roch (Sommerfeld, 1976). The stability factor is defined as the ratio of the mean sample shear strength corrected for normal pressure to the shear stress component parallel to the slope at a distinct point of the slope at the moment of a natural avalanche release. The shear strength was also measured with a shear frame. The stability factor given by Roch is about 2. This is indeed a very good agreement. But what happens if the measurements are performed with a different shear-frame size? Perla found a dependence of the measured shear strength of the sample volume or sample area: The larger the volume the lower the mean measured shear strength. This fact sustains the proposition that Perla measured brittle shear strength and at the same time that the shearframe area cannot be considered as a link unit. Besides this fact it has been shown in this paper that the ductile strength of snow depends strongly on the type of distribution of the strength of 
the links independent of the distribution width. The normal distribution applied by Sommerfeld for the link strength presumes a considerable probability for links of zero strength. This again sustains the conclusion that the chosen link size is not in agreement with the model rules. Therefore it seems that the above-mentioned agreement is accidental. The stability factor measured by Roch and its high relative standard deviation is likely to be at least in part a consequence of the method of sampling shear strength with the shear frame. Even if the shear strength of a thin lubricating layer could be determined correctly and simultaneously with the avalanche release, we do not know if we have really sampled the shear strength in the primary fracture volume.

\section{Conclusions}

Statistical models to estimate the strength of snow together with consistent definitions of the fundamental unit may lead to a better understanding of brittle and ductile strength of snow in terms of structural or stereological parameters.

\section{Agknowledgement}

I am indebted to B. Salm and M. de Quervain for valuable discussions.

MS. received 31 August 1977 and in revised form I6 November 1977

\section{REFERENCES}

Daniels, H. E. 1945. The statistical theory of the strength of bundles of threads. I. Proceedings of the Royal Society of London, Ser. A, Vol. 183, No. 995, p. 405-35.

Epstein, B. 1948. Statistical aspects of fracture problems. Fournal of Applied Physics, Vol. 19, No. 2, p. 140-47.

Gubler, H. 1976. Künstliche Auslösung von Lawinen durch Sprengungen. Mitteilungen des Eidg. Institutes für Schnee- und Lawinenforschung, No. 32.

Gubler, H. 1978. Determination of the mean number of bonds per snow grain and of the dependence of the tensile strength of snow on stereological parameters. Fournal of Glaciology, Vol. 20, No. 83, p. 329-4I. Kry, P. R. 1975[a]. Quantitative stereological analysis of grain bonds in snow. Fournal of Glaciology, Vol. 14,
No. 72, p. $467-77$.

Kry, P. R. 1975[b]. The relationship between the visco-elastic and structural properties of fine-grained snow. Journal of Glaciology, Vol. I4, No. 72, p. 479-500.

Salm, B. r971. On the rheological behavior of snow under high stresses. Contributions from the Institute of Low Temperature Science, Hokkaido University (Sapporo), Ser. A, No. 23.

Sommerfeld, R. A. 1973. Statistical problems in snow mechanics. U.S. Dept. of Agriculture. Forest Service. General Technical Report RM-3, p. 29-36.

Sommerfeld, R. A. r974. A Weibull prediction of the tensile strength-volume relationship in snow. Journal of Geophysical Research, Vol. 79, No. 23, p. $3353-56$.

Sommerfeld, R. A. I 976 . A correction factor for Roch's stability index of slab avalanche release. Fournal of Glaciology, Vol. 1 7, No. 75, p. 145-47.

Weibull, W. I939[a]. The phenomenon of rupture in solids. Ingeniörsvetenskapsakademiens Handlingar, Nr. I53. Weibull, W. I939[b]. A statistical theory of the strength of materials. Ingeniörsvetenskapsakademiens Handlingar,
Nr. I5 1 . 